\title{
A!
}

This is an electronic reprint of the original article.

This reprint may differ from the original in pagination and typographic detail.

Heikkila, T.T.; Massel, F.; Tuorila, J.; Khan, R.; Sillanpää, Mika A.

\section{Enhancing Optomechanical Coupling via the Josephson Effect}

Published in:

Physical Review Letters

DOI:

10.1103/PhysRevLett.112.203603

Published: $23 / 05 / 2014$

Document Version

Publisher's PDF, also known as Version of record

Please cite the original version:

Heikkila, T. T., Massel, F., Tuorila, J., Khan, R., \& Sillanpää, M. A. (2014). Enhancing Optomechanical Coupling via the Josephson Effect. Physical Review Letters, 112(20), 1-6. [203603].

https://doi.org/10.1103/PhysRevLett.112.203603

This material is protected by copyright and other intellectual property rights, and duplication or sale of all or part of any of the repository collections is not permitted, except that material may be duplicated by you for your research use or educational purposes in electronic or print form. You must obtain permission for any other use. Electronic or print copies may not be offered, whether for sale or otherwise to anyone who is not an authorised user. 


\title{
Enhancing Optomechanical Coupling via the Josephson Effect
}

\author{
T. T. Heikkilä, ${ }^{1,2}$ F. Massel, ${ }^{2,3}$ J. Tuorila, ${ }^{4}$ R. Khan, ${ }^{2}$ and M. A. Sillanpääa ${ }^{2,5}$ \\ ${ }^{1}$ Department of Physics and Nanoscience Center, University of Jyväskylä, P.O. Box 35 (YFL) FI-40014 University of Jyväskylä, Finland \\ ${ }^{2}$ Low Temperature Laboratory, Aalto University, P.O. Box 15100, FI-00076 Aalto, Finland \\ ${ }^{3}$ Department of Mathematics and Statistics, University of Helsinki, FI-00014 Helsinki, Finland \\ ${ }^{4}$ Department of Physics, University of Oulu, P.O. Box 3000, FI-90014 University of Oulu, Finland \\ ${ }^{5}$ Department of Applied Physics, Aalto University, P.O. Box 11100, FI-00076 Aalto, Finland
}

(Received 15 November 2013; published 23 May 2014)

\begin{abstract}
Cavity optomechanics is showing promise for studying quantum mechanics in large systems. However, the smallness of the radiation-pressure coupling is a serious hindrance. Here we show how the charge tuning of the Josephson inductance in a single-Cooper-pair transistor can be exploited to arrange a strong radiation-pressure-type coupling $g_{0}$ between mechanical and microwave resonators. In a certain limit of parameters, such a coupling can also be seen as a qubit-mediated coupling of two resonators. We show that this scheme allows reaching extremely high $g_{0}$. Contrary to the recent proposals for exploiting the nonlinearity of a large radiation-pressure coupling, the main nonlinearity in this setup originates from a cross-Kerr type of coupling between the resonators, where the cavity refractive index depends on the phonon number. The presence of this coupling will allow accessing the individual phonon numbers via the measurement of the cavity.
\end{abstract}

DOI: 10.1103/PhysRevLett.112.203603

Recent experiments on cavity optomechanical systems have shown how the parametric coupling between an electromagnetic (either optical or microwave) cavity and a mechanically vibrating resonator can be exploited to take the latter to its quantum mechanical ground state [1,2]. Such schemes rely on amplifying the intrinsically weak radiation-pressure coupling $g_{0}$ between the two systems via a strong pumping of the cavity, making the effective coupling between the systems linear. However, linearly coupled oscillators constitute a linear system lying in the correspondence limit, where quantum effects can be seen only in signal fluctuations $[3,4]$. Therefore, the emphasis of this research has shifted to the regime of strong radiationpressure coupling. There are many recent theoretical proposals of the ensuing dynamics of the system in the strong coupling regime [5-17], but reaching this regime in any system other than cold atom gases $[18,19]$ in practice is challenging $[20,21]$. The ultimate aim would be to make the bare radiation-pressure coupling of the order either of the frequency of the mechanical resonator $\omega_{m}$ or at least of the linewidth $\kappa$ of the cavity.

In this Letter we propose to use the nonlinearity of the Josephson effect to enhance the coupling between the vibrations and the electromagnetic field. The scheme involves a tripartite system consisting of a Josephson junction qubit, a microwave cavity, and a micromechanical resonator. Although previous works exist on coupling a qubit to both a cavity and a mechanical system [22-25], our work is, to our knowledge, the first where the system is considered as an optomechanical platform.

For representative superconducting circuit parameters [26], we find that the radiation-pressure coupling can be
PACS numbers: 42.50.Wk, 73.23.Hk, 81.07.Oj, 85.25.Cp

amplified by a large factor. We first show this by a simple Josephson inductance picture and then detail a SchriefferWolff-type approach where the effect is obtained as a systematic perturbation theory on the tripartite quantum system. Using this approach we also discuss the possible added mechanical and cavity damping due to the hybridization of the different parts of the system. Finally, we continue the perturbation theory to show that the nonlinear frequency shifts in this system are not primarily caused by the radiation-pressure coupling, but rather a cross-Kerrtype coupling.

Radiation pressure from Josephson inductance.-Here we take advantage of a charge qubit $[27,28]$, that is, a system of two small-capacitance Josephson junctions. This system is also known as a single-Cooper-pair transistor (SCPT), which is the picture that we first adopt. It behaves as a tunable inductance dependent on the mechanical displacement.

As marked by the dashed box in Fig. 1, the SCPT has junction capacitances $C_{1}, C_{2}$ and gate capacitance $C_{g 0}$, which give the charging energy of a single electron $E_{C}=e^{2} /\left[2\left(C_{g 0}+C_{1}+C_{2}\right)\right]$. The junctions have Josephson energies $E_{J i} \lesssim E_{C}$. Because of Coulomb blockade, the energy difference of having zero or one Cooper pair on the island can be tuned by the gate charge $n_{g 0}=V_{g} C_{g 0} / 2 e$. Moreover, Josephson tunneling mixes charge states into coherent superpositions of Cooper pair states [29].

In the most relevant limit $E_{C} \gg E_{J i}$, so that we concentrate on the two charge states closest to $n_{g 0}$, defining $\delta n_{g 0}=n_{g 0}-\operatorname{int}\left(n_{g 0}\right) \in[0,1]$ as the deviation of $n_{g 0}$ from the lower integer value. The Hamiltonian is [30] 


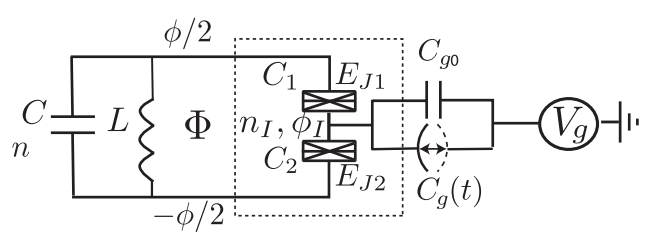

FIG. 1. Microwave optomechanical circuit considered here. The SCPT part is marked with a dashed box. The mechanical resonator couples via a time-dependent capacitance $C_{g}[x(t)]$.

$$
H_{\mathrm{SCPT}}=\sum_{j=1}^{3} B_{j} \sigma_{j} / 2
$$

where $\quad B_{1}=-\left(E_{J 1}+E_{J 2}\right) \cos (\phi / 2), \quad B_{2}=\left(E_{J 1}-\right.$ $\left.E_{J 2}\right) \sin (\phi / 2)$ and $B_{3}=4 E_{C}\left(1-2 \delta n_{g 0}\right)$ are the effective magnetic fields, $\sigma_{j}$ are Pauli matrices acting on the space spanned by the Cooper-pair charge states $\left|\operatorname{int}\left(n_{g}\right)\right\rangle$ and $\left|\operatorname{int}\left(n_{g}\right)+1\right\rangle$, and $\phi$ is the phase difference of the superconducting order parameters across the junction. The ground state energy is $E_{\mathrm{SCPT}}=-\sqrt{\sum_{j} B_{j}^{2}} / 2 \equiv-B / 2$.

Placing a Josephson junction inside an electromagnetic resonator (cavity) affects its total inductance via the Josephson inductance $L_{J}=\hbar^{2} /(2 e)^{2}\left[\partial_{\phi}^{2} E(\phi)\right]^{-1}$, where $E(\phi)$ is the energy of the junction. Using a SCPT instead of a single junction allows for controlling Josephson inductance via the modulation of the gate charge [26]. Here we consider what happens when the gate capacitor can vibrate, modulating the movable part of the gate capacitance $C_{g}(x, t)$ (see Fig. 1). The total gate charge is $n_{g}=n_{g 0}+x V_{g} \partial_{x} C_{g}$, where $x$ is the amplitude of mechanical vibrations. Along the dependence of the energy of the SCPT on both control parameters $n_{g}$ and $\phi$, the mechanical vibrations modulate the cavity eigenfrequency, and the resulting coupling is of the radiation-pressure type.

The above picture allows us to estimate the size of the radiation-pressure coupling. The cavity eigenfrequency $\omega_{c}=\left[\left(L|| L_{J}\right) C\right]^{-1 / 2}$ consists of the geometric and the Josephson inductances $L$ and $L_{J}$, respectively. The radiation-pressure coupling is, thus,

$$
g_{0} \equiv x_{\mathrm{ZP}} \frac{\partial \omega_{c}}{\partial x}=\frac{\omega_{c} x_{\mathrm{ZP}} \partial_{x} C_{g} V_{g}}{4 e} \frac{L L_{J}}{L+L_{J}} \partial_{n_{g}} L_{J}^{-1}
$$

Here $x_{\mathrm{ZP}}=\sqrt{\hbar /\left(2 m \omega_{m}\right)}$ is the zero-point motion amplitude for a mechanical resonator with effective mass $m$ and angular frequency $\omega_{m}$. Let us compare this with the coupling in the setup where the capacitance of the cavity is directly modulated $[1,4,31]$. In that case, $g_{0}^{d}=x_{\mathrm{ZP}} \partial_{x} C_{g} /$ $(2 C) \omega_{c}$. The ratio between these two couplings is

$$
\frac{g_{0}}{g_{0}^{d}}=\frac{C V_{g}}{2 e} \frac{L L_{J}}{L+L_{J}} \partial_{n_{g}} L_{J}^{-1}
$$

Choosing $L \approx L_{J}$ and noting that the factor $L_{J} \partial_{n_{q}} L_{J}^{-1}$ can be of the order of unity (see below), the optomechanical coupling can be amplified in this setting by the factor $C V_{g} /(2 e)$, about 4-6 orders of magnitude for typical experimental parameters [26,32].

It is now straightforward to obtain the radiation-pressure coupling from Eq. (3). For symmetric junctions, $E_{J}=$ $E_{J 1}=E_{J 2}$, we get

$$
\frac{g_{0}}{g_{0}^{d}}=\frac{C V_{g}}{2 e} \frac{8 E_{C}^{2} E_{J}^{2} l\left(1-2 \delta n_{g 0}\right)}{\left(4 \tilde{E}_{C}^{2}+E_{J}^{2}\right)\left(4 \sqrt{E_{J}^{2}+4 \tilde{E}_{C}^{2}}+E_{J}^{2} l\right)},
$$

where $\tilde{E}_{C}=E_{C}\left(1-2 \delta n_{g 0}\right)$ and $l=L(2 e)^{2} / \hbar^{2}$. We plot $g_{0}$ versus $\delta n_{g 0}$ in Fig. 2. The two-state approximation is generally valid for $E_{C} \gtrsim E_{J}$ as long as $n_{g 0}$ is not too close to an integer. For low $E_{J} / E_{C}, g_{0}$ contains a peak of width $\sim E_{J} / E_{C}$ with a maximum somewhat below the charge degeneracy point $\delta n_{g 0}=1 / 2$. For $l=1 / E_{J}$, the maximum resides at $\delta n_{g 0} \approx 1 / 2-0.18 E_{J} / E_{C}$ and is $\max _{\delta n_{00}} g_{0} \approx$ $0.32 g_{0}^{d} C V_{g} /(2 e) E_{C} / E_{J}$. The largest $g_{0}$ is thus obtained in the extreme charge qubit limit $E_{C} \gg E_{J}$, but because the range of gate charge values where this maximum is obtained is proportional to $E_{J} / E_{C}$, in practice it is preferable to choose $E_{J}$ not too far from $E_{C}$ to prevent gate charge fluctuations from masking the effect.

To make a numerical estimate of the resulting radiationpressure coupling, let us choose $(2 e)^{2} L / \hbar^{2}=E_{J}=E_{C} / 2$. For some representative values $C=50 \mathrm{fF}, V_{g}=10 \mathrm{~V}$, we would get $g_{0} / g_{0}^{d} \approx 10^{6}$. With the typical direct radiationpressure coupling $g_{0}^{d} /(2 \pi) \sim 10 \mathrm{~Hz}$ [33], we would hence get $g_{0} /(2 \pi) \sim 10 \mathrm{MHz}$, which is already of the order of typical $\omega_{m}$ and 2 orders of magnitude larger than a typical

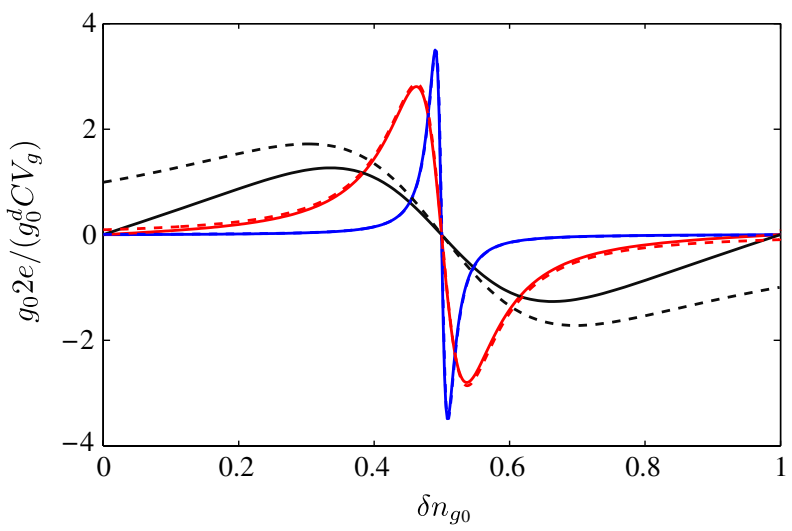

FIG. 2 (color online). Radiation-pressure coupling $g_{0}$ versus gate charge $\delta n_{g 0}$ for the case of equal Josephson couplings $\left(E_{J 1}=E_{J 2}\right)$, at flux $\Phi=0$ and for three different ratios $E_{J} / E_{C}=$ 0.05 (blue), 0.2 (red), and 1 (black), also denoted in the figure. The inductance $L=20 \hbar^{2} /\left(4 e^{2} E_{C}\right)$ in each curve. Solid lines show the results from the numerically obtained SCPT spectrum beyond the two-charge state restriction in Eq. (1), whereas the dashed lines are plotted from Eq. (4). 


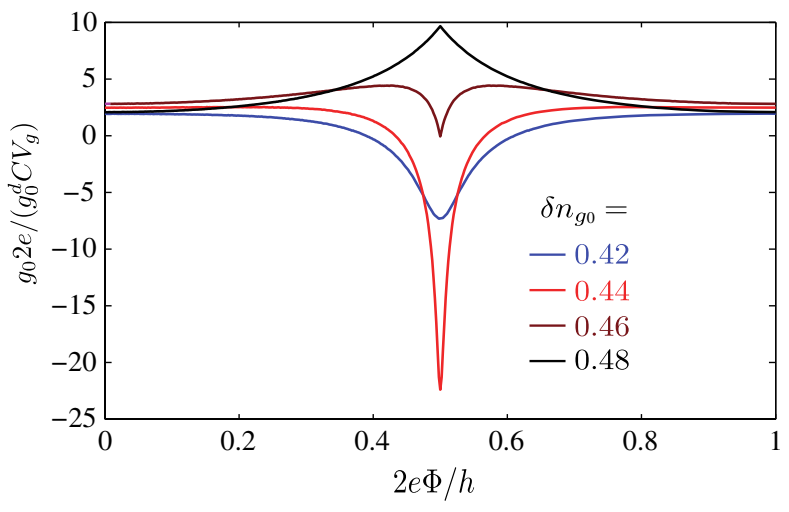

FIG. 3 (color online). Radiation-pressure coupling $g_{0}$ versus flux bias for a few values of the gate charge indicated in the plot for $E_{J 1}=E_{J 2}=0.2 E_{C}$ and $L=20 \hbar^{2} /\left(4 e^{2} E_{C}\right)$.

Al cavity linewidth $\kappa$ [1] below $100 \mathrm{mK}$. However, in practice the linewidths are affected by the charge noise of the qubit (see below).

In the presence of a flux $\Phi$ through the cavity loop, the average phase $\phi_{a}=\langle\phi\rangle$ is the phase that minimizes the total energy [34],

$$
\frac{\hbar^{2}}{8 e^{2} L}\left(\frac{2 e \Phi}{\hbar}-\phi\right)^{2}+E_{\mathrm{SCPT}}\left(\phi, n_{g 0}\right) .
$$

For a vanishing flux, $\phi_{a}=0$. It is then possible to tune the radiation-pressure coupling with the flux; see Fig. 3. Interestingly, such a flux tuning is stronger for smaller $E_{J}$, but the region of gate charges where $g_{0}$ is appreciable is again limited to a range proportional to $E_{J} / E_{C}$.

Another way to see why the coupling is boosted is because of the qubit nonlinearity. A mechanical resonator can be coupled to a linear cavity (not to a qubit as in the present work) by means of a voltage bias [35]. This coupling has a magnitude comparable to $g_{0}$. This coupling, however, is linear, and has few consequences between two linear resonators. Replacing the cavity by a qubit, however, turns the linear coupling into a longitudinal coupling, which has a strong influence on the energies.

Schrieffer-Wolff approach.-The Josephson inductance approach provides an intuitive picture of the physics. However, for a more rigorous treatment, we start from the general tripartite Hamiltonian and derive the optomechanical coupling by using the Schrieffer-Wolff transformation [36]. It consists of a unitary transformation which uncouples the high- and the low-energy states, leading to the definition of an effective low-energy Hamiltonian. Here, the high-energy states are represented by the qubit states while the low-energy ones are represented by cavity and mechanical oscillator modes. The ensuing effective low-energy Hamiltonian is thus described in terms of effective cavity and mechanical oscillator modes. Note that both this and the above Josephson inductance approach are valid only in the dispersive limit, where $\hbar \omega_{c / m} \ll|B|$.

We express the electromagnetic energies of the circuit in Fig. 1 in terms of the phases $\phi$ and $\phi_{I}$ with conjugate charges $2 e n$ and $2 e n_{I}$ ( $n$ and $n_{I}$ denote the number of Cooper pairs and $I$ points to the SCPT island). Then we use the fact that $e^{i \phi_{I}}$ is a ladder operator for charge $n_{I}$ [29], assume that changes of $\phi$ with respect to $\phi_{a}$ are small compared to $2 \pi$ (in the opposite limit the dynamics of the system is quite complicated, see for example Ref. [37]), and define $\phi-\phi_{a}=\phi_{0}\left(c^{\dagger}+c\right)$ with the conjugate variable $n=i \sqrt{\hbar /\left(8 e^{2} Z_{0}\right)}\left(c^{\dagger}-c\right)$. Here, $Z_{0}=\sqrt{L / C}$ and the phase zero-point fluctuation is $\phi_{0}=\sqrt{2 e^{2} Z_{0} / \hbar}$. We then write the resulting Hamiltonian for the system in the charge basis as above. With a similar quantization of the mechanical part of the Hamiltonian, we get (see detailed derivation in Ref. [38])

$$
\begin{aligned}
H= & H_{\mathrm{SCPT}}+\hbar \omega_{c}^{0} c^{\dagger} c+\hbar \omega_{m}^{0} a^{\dagger} a+g_{m} \sigma_{3}\left(a^{\dagger}+a\right) \\
& +\left(g_{q 1} \sigma_{1}+g_{q 2} \sigma_{2}\right)\left(c^{\dagger}+c\right)^{2} \\
& +\left(g_{c 1} \sigma_{1}+g_{c 2} \sigma_{2}\right)\left(c^{\dagger}+c\right) .
\end{aligned}
$$

Here $\omega_{c}^{0}=(L C)^{-1 / 2}$ and $\omega_{m}^{0}$ are the eigenfrequency of the bare $L C$ oscillator and of the bare mechanics, respectively, $B_{j}$ are as above, and

$$
\begin{gathered}
g_{m}=-\frac{4 E_{C} x_{\mathrm{ZP}} \partial_{x} C_{g} V_{g}}{2 e}, \\
g_{q 1}=\frac{e^{2} Z_{0}}{8 \hbar}\left(E_{J 1}+E_{J 2}\right) \cos \left(\phi_{a} / 2\right), \\
g_{q 2}=\frac{e^{2} Z_{0}}{8 \hbar}\left(E_{J 2}-E_{J 1}\right) \sin \left(\phi_{a} / 2\right), \\
g_{c 1}=\sqrt{\frac{e^{2} Z_{0}}{8 \hbar}}\left(E_{J 1}+E_{J 2}\right) \sin \left(\phi_{a} / 2\right), \\
g_{c 2}=\sqrt{\frac{e^{2} Z_{0}}{8 \hbar}}\left(E_{J 1}-E_{J 2}\right) \cos \left(\phi_{a} / 2\right) .
\end{gathered}
$$

The system is thus composed of two resonators with frequencies $\omega_{c}^{0}$ and $\omega_{m}^{0}$ coupled to a common qubit. We note that the order of magnitude of the cavity couplings satisfies $g_{q j} \sim g_{c j}^{2} / E_{J} \gg g_{m}$. Below we limit ourselves to the case of a symmetric system $E_{J 1}=E_{J 2}$, in which case $B_{2}=g_{q 2}=g_{c 2}=0$. The full results are given in Ref. [38].

The full Schrieffer-Wolff transformation diagonalizing the qubit part of the system is quite complicated. However, in the dispersive limit $\hbar \omega_{c, m} \ll|B|$, and where all couplings are smaller than the difference $|B|-\hbar \omega_{c, m}$, it is enough to diagonalize the qubit treating the oscillator coordinates as scalars. Assuming that this effective qubit 
stays in its ground state, we may replace $\sigma_{3} \rightarrow-1$. Expanding in the coupling constants, we get a preliminary form of the Hamiltonian:

$$
\begin{aligned}
H_{\mathrm{eff}}= & \hbar \omega_{c}^{0} c^{\dagger} c+\hbar \omega_{m}^{0} a^{\dagger} a+\alpha_{c} \hat{x}_{c}+\alpha_{m} \hat{x}_{m}+\hbar g_{c m} \hat{x}_{c} \hat{x}_{m} \\
& +\hbar g_{S c} \hat{x}_{c}^{2}+\hbar g_{S m} \hat{x}_{m}^{2}+\hbar g_{r p} \hat{x}_{c}^{2} \hat{x}_{m} .
\end{aligned}
$$

Here $\hat{x}_{c} \equiv\left(c^{\dagger}+c\right), \hat{x}_{m} \equiv\left(a^{\dagger}+a\right)$, and the coefficients are $\alpha_{c}=-B_{1} g_{c 1} / B, \alpha_{m}=-B_{3} g_{m} / B, \hbar g_{c m}=2 B_{3} B_{1} g_{c 1} g_{m} / B^{3}$, and

$$
\begin{aligned}
& \hbar g_{S m}=-\frac{B_{1}^{2} g_{m}^{2}}{B^{3}}, \quad \hbar g_{S c}=-\frac{B_{3}^{2} g_{c 1}^{2}+B^{2} B_{1} g_{q 1}}{B^{3}}, \\
& \hbar g_{r p}=\frac{2 B_{3} g_{m}\left[B^{2} B_{1} g_{q 1}+\left(B_{3}^{2}-2 B_{1}^{2}\right) g_{c 1}^{2}\right]}{B^{5}} .
\end{aligned}
$$

The first two terms in Eq. (8) are the Hamiltonians for the bare oscillators, the next two are qubit-induced static forces on them (neglected below), the term proportional to $g_{c m}$ is a linear coupling between the oscillators, and the terms with coefficients $g_{S c}$ and $g_{S m}$ are the cavity and mechanical Stark shifts $[25,32,40]$. Finally, $g_{r p}$ denotes an intermediate expression of a radiation-pressure-type coupling. We diagonalize the individual oscillator Hamiltonians by the Bogoliubov transformation, introducing $c=\cosh \left(\theta_{c}\right) d+$ $\sinh \left(\theta_{c}\right) d^{\dagger}$ and $a=\cosh \left(\theta_{m}\right) b+\sinh \left(\theta_{m}\right) b^{\dagger}$, with $\theta_{c / m}=$ $-\operatorname{arctanh}\left[\left(2 g_{S c / m}\right) /\left(\hbar \omega_{c / m}^{0}+2 g_{S c / m}\right)\right] / 2$. This yields the effective frequencies $\omega_{c}=\sqrt{\omega_{c}^{0}\left(\omega_{c}^{0}+4 g_{S c}\right)}$ and $\omega_{m}=$ $\sqrt{\omega_{m}^{0}\left(\omega_{m}^{0}+4 g_{S m}\right)}$ for the cavity and the vibrations, respectively. The linear coupling $g_{c m}$ has little effect, and is neglected. Including the Bogoliubov transformations in the radiation-pressure coupling and assuming $g_{r p} x_{m} \ll$ $\omega_{c}$ yields a coupling between the effective mechanics and cavity of the form $\hbar g_{0} d^{\dagger} d\left(b^{\dagger}+b\right)$. Here $g_{0}=$ $2 g_{r p}\left(\omega_{c}^{0} / \omega_{c}\right)\left(\omega_{m}^{0} / \omega_{m}\right)^{1 / 2}$ [41] is the radiation-pressure coupling. These results then coincide with those of the above Josephson inductance approach, except for the renormalization due to mechanical Stark shift $\left(\omega_{m}^{0} / \omega_{m}\right)^{1 / 2}$ that is not captured by the latter.

Quantum nonlinearities. - The possibility of obtaining a large Josephson-enhanced radiation-pressure coupling $g_{0}$ implies a good prospect of reaching the "quantum regime" of optomechanics, where $g_{0}$ becomes at least of the order of the cavity linewidth $\kappa$. In this regime it should be possible to observe nonlinearities directly in the spectrum. The frequency shift is proportional to $g_{0}^{2} / \omega_{m}$ [9], and is of the order $\sim g_{c j}^{4} g_{m}^{2} \sim g_{q j}^{2} g_{m}^{2}$. However, the qubit-mediated coupling has another nonlinearity that gives rise to a frequency shift in the mechanics and shows up at a lower order. It can be understood as the change in the photon Stark shift (which depends on the qubit level splitting) due to the phonon-driven qubit Stark shift, and it implies a term of the form $c^{\dagger} c a^{\dagger} a$. It is thus of the form of the cross-Kerr effect between the two resonators. In the perturbation series with respect to the couplings $g_{c j}, g_{q j}$ and $g_{m}$, such a term would be of the order of $g_{c j}^{2} g_{m}^{2} \sim g_{q j} g_{m}^{2}$. In the rotating wave approximation we get the term $H_{\mathrm{CK}}=\hbar g_{\mathrm{CK}} d^{\dagger} d b^{\dagger} b$, with

$$
\frac{g_{\mathrm{CK}}}{g_{0}}=\frac{R_{Q}}{Z_{0}} \frac{\hbar g_{0}}{E_{J}} \frac{\left[4 \tilde{E}_{c}^{2}\left(8 E_{s}+E_{J}^{2} l\right)-E_{J}^{2}\left(4 E_{s}+E_{J}^{2} l\right)\right]}{2 \pi \tilde{E}_{c}^{2} E_{J} \sqrt{4+\frac{E_{J}^{2}}{E_{s}}}},
$$

where $R_{Q}=h /(2 e)^{2}$ and $E_{s}=\sqrt{4 \tilde{E}_{C}^{2}+E_{J}^{2}}$. The total effective optomechanical Hamiltonian is thus

$$
H_{\text {eff }}=\hbar \omega_{c} d^{\dagger} d+\hbar \omega_{m} b^{\dagger} b+\hbar g_{0} d^{\dagger} d\left(b^{\dagger}+b\right)+H_{\mathrm{CK}} .
$$

Whereas the response with sideband driving is dominated by the large $g_{0}$, the nonlinear frequency shifts are mainly due to the term $g_{\mathrm{CK}}$ [see the spectrum of Eq. (10) in Ref. [38])]. For example, for $1 / l \approx E_{J} \approx \tilde{E}_{c}$, where the radiation-pressure coupling $g_{0}$ is appreciable, we get $g_{\mathrm{CK}} / g_{0} \approx 5 \hbar g_{0} R_{Q} /\left(E_{J} Z_{0}\right)$. With $g_{0} \approx 5 \mathrm{MHz}, E_{J} / \hbar \approx$ $10 \mathrm{GHz}$, and $Z_{0} \approx R_{Q} / 100$, we would hence get an appreciable nonlinearity, $g_{\mathrm{CK}} \approx 0.25 g_{0}$. Moreover, close to $n_{g 0}=1 / 2$, the radiation-pressure term vanishes whereas the cross-Kerr term is finite. The Hamiltonian becomes particularly simple, as the coupling commutes with the rest of the Hamiltonian. As a result, the cavity frequency is shifted by the number of quanta in the mechanical resonator. Such a shift could be used for a direct detection or creation of the Fock states in the mechanical resonator.

Effect of qubit-mediated dissipation.-Since the qubit and the oscillators are generally hybridized up to a significant amount, it is important to consider the effect of qubit energy relaxation on that of the oscillators. This can be analyzed with the Schrieffer-Wolff approach, but now applying the transformation only to the qubit-oscillator part of the setup [38]. We find that the rates for relaxation or excitation of the mechanical resonator due to the qubit dissipation satisfy

$$
\frac{\gamma_{\mathrm{rel} / \mathrm{exc}}}{\gamma_{\mathrm{rel} / \mathrm{exc}}^{q}}=\frac{2 g_{m}^{2} B_{1}^{2}}{B^{4}} \frac{\sum_{j} \lambda_{j}^{2} S_{j}\left( \pm \omega_{m}\right)}{\sum_{j} \lambda_{j}^{2} S_{j}( \pm B)} .
$$

Here $\gamma_{\text {rel,exc }}^{q}$ are the bare qubit relaxation/excitation rates, $\lambda_{j}$ is the coupling between the qubit and the bath oscillator $j$, and $S_{j}(\omega) \equiv \int d t e^{i \omega t}\left\langle\left[b_{j}^{\dagger}(t)+b_{j}(t)\right]\left[b_{j}^{\dagger}(0)+b_{j}(0)\right]\right\rangle$ is the correlator of the qubit bath, chosen diagonal for convenience. For an equilibrium bath at temperature $T_{b}$, these satisfy a detailed balance relation $\gamma_{\text {rel }} / \gamma_{\text {exc }}=S_{j}\left(\omega_{m}\right) /$ $S_{j}\left(-\omega_{m}\right)=\exp \left[\hbar \omega_{m} /\left(k_{B} T_{b}\right)\right]$. Using the fluctuation-dissipation relation with a frequency independent susceptibility for the bath correlator (i.e., quantum noise increasing linearly with an increasing frequency), we would then get at $k_{B} T_{b} \lesssim \hbar \omega_{m}$ the induced mechanical dissipation rate 
$\gamma_{\text {rel }} \approx g_{m}^{2} \omega_{m} B_{1}^{2} \gamma_{\text {rel }}^{q} / B^{5}$, which is likely quite small in practical systems. However, in the case of charge qubits, one should consider $1 / f$ (flicker) noise, i.e., noise increasing linearly with a decreasing frequency. In this case this relation changes roughly to $\gamma_{\text {rel }} \approx g_{m}^{2} B_{1}^{2} /\left(B^{3} \omega_{m}\right) \gamma_{q \text {,rel }}$, which may already become relevant compared to the intrinsic oscillator dissipation at large values of $g_{m}$.

In the case of the cavity, we get similar effects on relaxation and excitation rates by replacing $g_{m}$ with $g_{c 1}$ or $g_{c 2}, B_{1}$ by $B_{3}$, and $\omega_{m}$ by $\omega_{c}$. However, a more relevant effect is likely due to pure cavity dephasing seen by a flickering of the cavity frequency due to low-frequency background charge fluctuations in the qubit. As analyzed in Ref. [38], the rate for this process in the case $E_{J 1}=E_{J 2}$ and $\phi_{a}=0$ is $\gamma_{\phi} \sim g_{q 1}^{2} \gamma_{\phi}^{q} / B^{2}$, where $\gamma_{\phi}^{q}$ is the pure dephasing rate of the qubit. At the optimal operation point, this is of the order of $e^{4} Z_{0}^{2} \gamma_{\phi}^{q} /\left(16 \hbar^{2}\right)$. For example, using $\gamma_{\phi}^{q}=$ $500 \mathrm{MHz}$ [42] and $Z_{0} \approx 500 \Omega$, the corresponding added cavity dephasing rate would be of the order of $0.5 \mathrm{MHz}$, which is already larger than the intrinsic $\kappa$. Nevertheless, as $g_{0} / \gamma_{\phi} \propto V_{g}$, this does not hinder reaching the limit of strong optomechanical coupling.

Note that the above approach is valid as long as the cavity photon number $n_{c}$ is not too large, such that phase fluctuations $\phi_{0} \sqrt{n_{c}}$ are small compared to $2 \pi$. Typical values are $\phi_{0} \sim 0.1, \ldots, 0.5$, depending on the cavity impedance. At the upper end of the scale there are nonlinear corrections to energy of $10 \%$ already at $n_{c} \sim 1$. In spite of the low linear regime, because of the large $g_{0}$, optomechanical phenomena are overwhelming already at photon numbers $n_{c} \approx 1$.

In summary, we have presented a realizable scheme for boosting the optomechanical radiation-pressure coupling by several orders of magnitude. This gives the possibility of approaching the previously elusive single-photon strong coupling limit of optomechanics. Our predictions can be readily tested in state-of-the-art circuit optomechanical devices.

We acknowledge fruitful discussions with Pertti Hakonen, Florian Marquardt, and Sorin Paraoanu. This work was supported by the Academy of Finland, the European Research Council (Grants No. 240362Heattronics, No. 240387-NEMSQED, and MPOES) and the EU-FP 7 INFERNOS (Grant No. 308850) and MICROKELVIN (Grant No. 228464) programs.

[1] J. D. Teufel, T. Donner, D. Li, J. W. Harlow, M. S. Allman, K. Cicak, A. J. Sirois, J. D. Whittaker, K. W. Lehnert, and R. W. Simmonds, Nature (London) 475, 359 (2011).

[2] J. Chan, T. P. M. Alegre, A. H. Safavi-Naeini, J. T. Hill, A. Krause, S. Gröblacher, M. Aspelmeyer, and O. Painter, Nature (London) 478, 89 (2011).
[3] A. H. Safavi-Naeini, J. Chan, J. T. Hill, Thiago P. Mayer Alegre, A. Krause, and O. Painter, Phys. Rev. Lett. 108, 033602 (2012).

[4] F. Massel, T. T. Heikkilä, J. M. Pirkkalainen, S. U. Cho, H. Saloniemi, P. J. Hakonen, and M. A. Sillanpää, Nature (London) 480, 351 (2011).

[5] S. Mancini, V. I. Man'ko, and P. Tombesi, Phys. Rev. A 55, 3042 (1997).

[6] S. Bose, K. Jacobs, and P. L. Knight, Phys. Rev. A 56, 4175 (1997).

[7] M. Ludwig, B. Kubala, and F. Marquardt, New J. Phys. 10, 095013 (2008).

[8] A. Nunnenkamp, K. Børkje, and S. M. Girvin, Phys. Rev. Lett. 107, 063602 (2011).

[9] P. Rabl, Phys. Rev. Lett. 107, 063601 (2011).

[10] J. Qian, A. A. Clerk, K. Hammerer, and F. Marquardt, Phys. Rev. Lett. 109, 253601 (2012).

[11] X-W. Xu, H. Wang, J. Zhang, and Y. X. Liu, Phys. Rev. A 88, 063819 (2013).

[12] A. Nunnenkamp, K. Børkje, and S. M. Girvin, Phys. Rev. A 85, 051803 (2012).

[13] K. Stannigel, P. Komar, S. J. M. Habraken, S. D. Bennett, M. D. Lukin, P. Zoller, and P. Rabl, Phys. Rev. Lett. 109, 013603 (2012).

[14] G.-F. Xu and C. K. Law, Phys. Rev. A 87, 053849 (2013).

[15] A. Kronwald and F. Marquardt, Phys. Rev. Lett. 111, 133601 (2013).

[16] U. Akram, W. P. Bowen, and G. J. Milburn, New J. Phys. 15, 093007 (2013).

[17] J.-Q. Liao and F. Nori, Phys. Rev. A 88, 023853 (2013).

[18] K. W. Murch, K. L. Moore, S. Gupta, and D. M. StamperKurn, Nat. Phys. 4, 561 (2008).

[19] F. Brennecke, S. Ritter, T. Donner, and T. Esslinger, Science 322, 235 (2008).

[20] J. Chan, A. H. Safavi-Naeini, J. T. Hill, S. Meenehan, and O. Painter, Appl. Phys. Lett. 101, 081115 (2012).

[21] A. Xuereb, C. Genes, and A. Dantan, Phys. Rev. Lett. 109, 223601 (2012).

[22] C. P. Sun, L. F. Wei, Y. X. Liu, and F. Nori, Phys. Rev. A, 73, 022318 (2006).

[23] E. Buks, S. Zaitsev, E. Segev, B. Abdo, and M. P. Blencowe, Phys. Rev. E 76, 026217 (2007).

[24] J. Zhang, Y. X. Liu, and F. Nori, Phys. Rev. A 79, 052102 (2009).

[25] J. M. Pirkkalainen, S. U. Cho, J. Li, G. S. Paraoanu, P. J. Hakonen, and M. A. Sillanpää, Nature (London) 494, 211 (2013).

[26] M. A. Sillanpää, L. Roschier, and P. J. Hakonen, Phys. Rev. Lett. 93, 066805 (2004).

[27] Y. Nakamura, Yu. A. Pashkin, and J.S. Tsai, Nature (London) 398, 786 (1999).

[28] D. Vion et al., Science 296, 886 (2002).

[29] Yu. Makhlin, G. Schön, and A. Shnirman, Rev. Mod. Phys. 73, 357 (2001).

[30] We use the convention that the quantities related to qubits ( $B_{j}$ fields and couplings) are in units of energy, whereas those related only to oscillators are in units of frequency.

[31] C. A. Regal, J. D. Teufel, and K. W. Lehnert, Nat. Phys. 4, 555 (2008). 
[32] M. D. LaHaye, J. Suh, P. M. Echternach, K. C. Schwab, and M. L. Roukes, Nature (London) 459, 960 (2009).

[33] J. Sulkko, M. A. Sillanpää, P. Häkkinen, L. Lechner, M. Helle, A. Fefferman, J. Parpia, and P. J. Hakonen, Nano Lett. 10, 4884 (2010).

[34] Typically there is a unique minimum, but for a complicated form of $E_{\mathrm{SCPT}}(\phi)$, there may be more than one of them. In that case we choose the global minimum. The presence of multiple minima would show up as hysteresis.

[35] L. Tian, Phys. Rev. B 79, 193407 (2009).

[36] S. Bravyi, D. DiVincenzo, and D. Loss, Ann. Phys. (Amsterdam) 326, 2793 (2011).

[37] V. Gramich, B. Kubala, S. Rohrer, and J. Ankerhold, Phys. Rev. Lett. 111, 247002 (2013).

[38] See the Supplemental Material at http://link.aps.org/ supplemental/10.1103/PhysRevLett.112.203603 for further details of the radiation pressure coupling, additional damping due to hybridization, the cross-Kerr coefficient, and a description of the spectrum of Eq. (10). It also includes Ref. [39].

[39] T. T. Heikkilä, The Physics of Nanoelectronics (Oxford University Press, Oxford, 2013).

[40] D. I. Schuster, A. Wallraff, A. Blais, L. Frunzio, R. S. Huang, J. Majer, S. M. Girvin, and R. J. Schoelkopf, Phys. Rev. Lett. 94, 123602 (2005).

[41] Note that the corrections $\left(\omega_{c}^{0} / \omega_{c}\right)$ and $\left(\left(\omega_{m}^{0} / \omega_{m}\right)^{1 / 2}\right)$ coming from the Bogoliubov transformation are formally of a higher order than assumed in the coupling expansion. The first term is necessary to properly account for the effect of the Josephson inductance in the limit $L_{J} \lesssim L$.

[42] D. Gunnarsson, J. Tuorila, A. Paila, J. Sarkar, E. Thuneberg, Yu. Makhlin, and P. Hakonen, Phys. Rev. Lett. 101, 256806 (2008). 